Special Issue of the 8th International Advances in Applied Physics and Materials Science Congress (APMAS 2018)

\title{
Multi-Analytical Study of Degradation Processes in Perovskite Films for Optoelectronic Applications
}

\author{
V. Travkin*, G. Pakhomov, P. Yunin and M. Drozdov \\ Institute for Physics of Microstructures of Russian Academy of Sciences (IPM RAS), \\ Nizhny Novgorod, Russian Federation
}

\begin{abstract}
The combination of three instrumental techniques: optical spectroscopy/microscopy, X-ray diffraction and mass-spectrometry (ToF-SIMS with depth profiling) was used to analyze the time, temperature and light induced degradation in thin films of the perovskite-type materials. Hybrid perovskite $\left(\mathrm{MAPbI}_{3}\right)$ and inorganic perovskite $\left(\mathrm{CsPbI}_{3}\right)$ films were obtained by a thermal vacuum evaporation and subjected to illumination by the continuous and monochromatic light. Photoelectrical measurements were carried out on the multilayer devices, in which perovskite was sandwiched between $\mathrm{CuI}$ hole transporting and $\mathrm{C}_{60}$ electron transporting layers.
\end{abstract}

DOI: 10.12693/APhysPolA.135.1039

PACS/topics: perovskite, morphology, vacuum deposition, photovoltaic cells, degradation

\section{Introduction}

Recently, we investigated the planar hybrid heterojunction $p-\mathrm{CuI} / n-\mathrm{C}_{60}$ for the semitransparent photovoltaic cells [1]. In the present work, we introduced the hybrid perovskite in this junction, so that $\mathrm{CuI}$ and $\mathrm{C}_{60}$ form an envelope of the hole- and electron-transporting layer, respectively. Perovskite (e.g., $\mathrm{MAPbI}_{3}, \mathrm{CsPbI}_{3}$ ) itself is a widely used in the photovoltaics due to unique electro-physical properties, however, performance of the perovskite-based cells is dramatically affected by the perovskite layer structure that is, in turn, determined by the processing conditions (substrate temperature, deposition rate, under- and overlying functional layers, etc.) [2, 3]. Therefore, the challenging issue is the proper adaptation of functional layer materials to the deposition techniques used for fabrication of the multilayer structure in order to minimize the degradation. Hereinafter, the deposition technique is thermal vacuum evaporation for all the materials used.

\section{Experimental procedures}

The cuprous iodide $\mathrm{CuI}(99.999 \%)$ and buckminsterfullerene $\mathrm{C}_{60}(99.9 \%)$ were used to form the charge transporting layers adjacent to the main perovskite photoabsorber. Glass substrates covered with a transparent indium tin oxide ITO (Aldrich, $25 \Omega / \mathrm{sq}$ ) and thin-film (semitransparent) silver $(99.9 \% \mathrm{SBRF})$ layer served as the bottom and top electrodes, respectively. Bathocuproine $\mathrm{BCP}$ and molybdenum trioxide $\mathrm{MoO}_{3}$ were used as the buffer layers materials. The functional layers were deposited sequentially on various substrates

*corresponding author; e-mail: trav@ipmras.ru using a VUP-5M vacuum station in the thermal evaporation mode at the pressure $<10^{-6}$ Torr in the following order: $\mathrm{MoO}_{3}(3 \mathrm{~nm}) / \mathrm{CuI}(15 \mathrm{~nm}) / \mathrm{MAPbI}_{3}(200 \mathrm{~nm}) / \mathrm{C}_{60}$ $(35 \mathrm{~nm}) / \mathrm{BCP}(8 \mathrm{~nm}) / \mathrm{Ag}(20 \mathrm{~nm})$. The thickness, density and morphology of the perovskite layers were studied by quartz crystal microbalancing, X-ray diffraction and reflectometry, XRD + XRR (Bruker D8 Discover diffractometer) and white-light interferometry, WLI (TalySurf CCI2000 profilometer). The time-of-flight secondary ion mass spectrometry (ToF-SIMS) experiments were carried out in a TOFSIMS5 spectrometer as described previously [4]. The optical transmission of samples in the range of 350-900 nm was analyzed with a FireFly 4000 spectrometer. The $J-V$ measurements were done using a Keithley SCS-4200 parameter analyzer. A mirror halogen lamp providing the average incident power of $25 \mathrm{~mW} / \mathrm{cm}^{2}$ and a MDR-41 LOMO monochromator were used for illumination. During measurements, the samples were kept into a shielded chamber filled with the ultrapure Ar to minimize the atmospheric effects $\left(\mathrm{O}_{2}, \mathrm{H}_{2} \mathrm{O}\right)$.

\section{Results and discussion}

Figure 1a shows the dynamic $J-V$ plots of the multilayer cell under the white light illumination. In Fig. 1b, a KC-10 cut-off filter was inserted between the light source and sample, thus irradiation with wavelengths shorter than $520 \mathrm{~nm}$ was damped. In both cases, the prolonged illumination leads to the stepwise degradation of characteristics. However, while the filtered light causes rather a decrease in $U_{o c}$ (by $\sim 0.2 \mathrm{~V}$ after $2 \mathrm{~h}$ exposure), the continuous white light causes both a weak decrease in $U_{o c}$ and dramatic drop of $J_{s c}$ (by more than 2 orders of magnitude).

To prove whether the Joule heat produced by illumination or incident photons are responsible for these changes, the cells were exposed to a low-intensity monochromatic light of $497 \pm 3 \mathrm{~nm}$. As seen from Fig. 1c, the degradation picture in such a case is similar to that induced by 

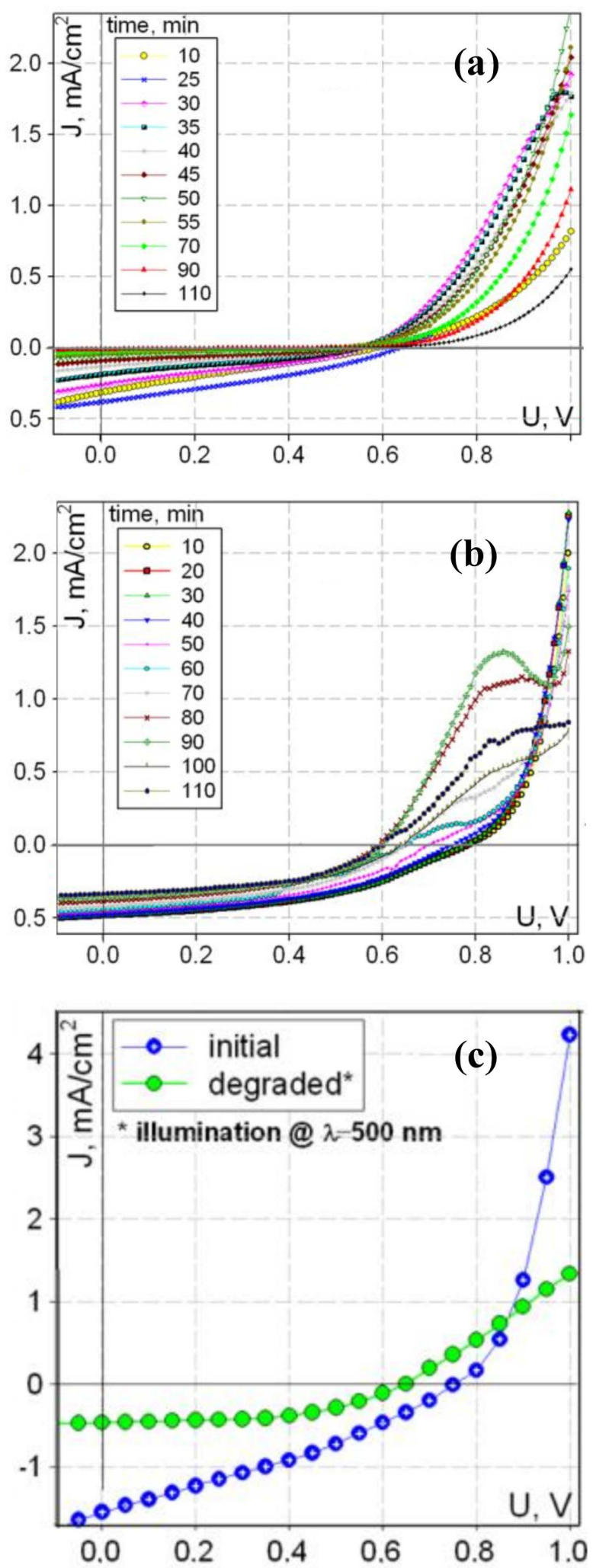

Fig. 1. $J-V$ plots for the cells glass $/ \mathrm{ITO} / \mathrm{MoO}_{3} /$ $\mathrm{CuI} / \mathrm{MaPbI}_{3} / \mathrm{C}_{60} / \mathrm{BCP} / \mathrm{Ag}$ : (a) illumination with continuous white light; (b) illumination through a filter cutting the short wavelength light; (c) illumination with a low-intensity monochromatic light of $500 \mathrm{~nm}$ during $20 \mathrm{~h}$ in the inert atmosphere.
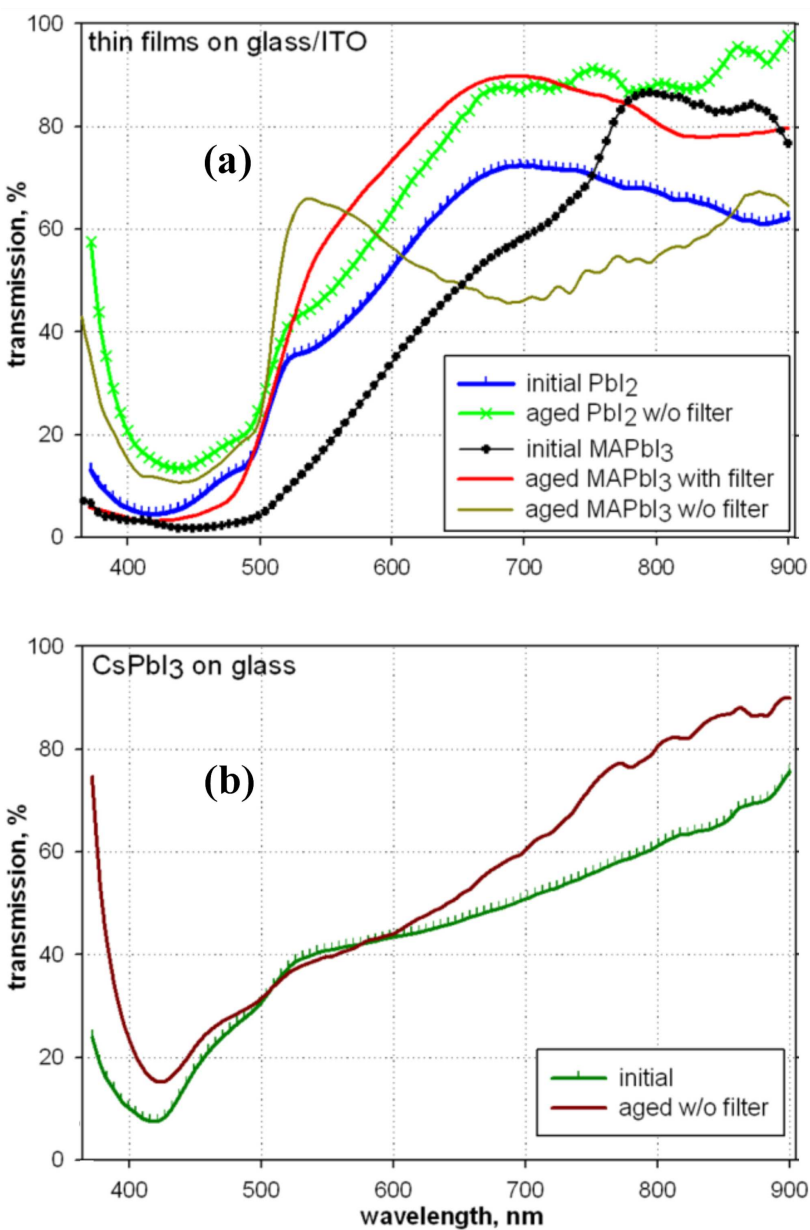

Fig. 2. Optical transmission spectra of (a) $\mathrm{MAPbI}_{3}$, $\mathrm{PbI}_{2}$ and (b) $\mathrm{CsPbI}_{3}$ films.

the unfiltered (continuous) light. This suggests that the photodestruction dominates over the thermodestruction even if the perovskite material is kept under the inert conditions [5]. It should be noted that forward and reserve bias $J-V$ dependences of degraded samples (not shown) do not coincide, possibly due to the presence of ionic component in the electrical conductivity.

$\mathrm{XRD}$, optical transmission and microscopic studies were performed to interpret the trend observed in the $J-V$ plots. Tang et al. [5] reported that $\mathrm{MAPbI}_{3}$ is always stable under illumination if kept under the inert atmosphere. However, even if we "aged" a $\mathrm{MAPbI}_{3}$ film on glass/ITO (blank sample) in the inert atmosphere by exposure to the white light for hundreds minutes, relative intensity of the $\mathrm{MAPbI}_{3}$ peaks in the XRD spectra decreases, whereas intensity of peaks related to the $\mathrm{PbI}_{2}$ phase increases.

Transparency of the strongly degraded $\mathrm{PbI}_{2}$ films tends to increase in the near infrared, but the overall shape of the optical spectrum unaltered with aging - Fig. 2a. Absorption of photons by the hybrid perovskite $\mathrm{MAPbI}_{3}$ suffers from aging even with a cut-off filter: the films become significantly more transparent at wavelengths 
longer than $500 \mathrm{~nm}$ and up to $800 \mathrm{~nm}$. Aging without filter leads to the further degradation of the spectral shape in this range - Fig. 2a. Similarity of spectra of the slightly degraded $\mathrm{MAPbI}_{3}$ film and the initial $\mathrm{PbI}_{2}$ film points to the increasing content of inorganic component in the perovskite structure.

Next, $\mathrm{MAPbI}_{3}$ was aged as a part of the multilayer photovoltaic cell (see, Fig. 1) over $2 \mathrm{~h}$ with and without a KC-10 optical filter with a stepwise monitoring of XRD pattern. When the sample was aged without filter, the XRD peaks related to $\mathrm{MAPbI}_{3}$ decrease, while the peaks of the $\mathrm{PbI}_{2}$ (001) phase grow up. When aging was carried out using the cut-off optical filter, no significant changes in the crystalline structure are detected. Therefore, degradation processes in the bulk of the hybrid perovskite are essentially due to the absorbed photons in the range below $520 \mathrm{~nm}$. Comparison of the optical and XRD studies indicates that the perovskite structure deteriorates starting from the re-texturization of the layer and the partial photodestruction of $\mathrm{PbI}_{2}$ (decomposition to metallic $\mathrm{Pb}$ and $\mathrm{I}_{2}[6]$ ), but dominating process is the rapid accumulation (segregation) of $\mathrm{PbI}_{2}$ phase.

In contrast to the hybrid perovskite, fully inorganic $\mathrm{CsPbI}_{3}$ films (on glass) do not degrade under illumination, whatever the atmosphere $(c f .-[3])$. It is confirmed by the minor changes in the optical transmission spectra of aged samples (Fig. 2b) and by the absence of alterations in the surface morphology of $\mathrm{CsPbI}_{3}$. So, the r.m.s surface roughness of the fresh made $\mathrm{MAPbI}_{3}$ film measured by WLI on the scanning area of $0.9 \times 0.9 \mathrm{~mm}^{2}$ is $2.2 \mathrm{~nm}$. Aging without filter increases this value to $3.4 \mathrm{~nm}$, whereas after aging with filter roughness increases only to $2.5 \mathrm{~nm}$. At the same time, the surface roughness of the $\mathrm{CsPbI}_{3}$ film remains equal to $2.4 \mathrm{~nm}$ after any kind/time of aging.

ToF-SIMS analysis with an accurate depth profiling was carried out for both negative and positive secondary ions characteristic of each component of the structure, including the bulk substrate. For the intact samples, the chemical interface is less abrupt when $\mathrm{MAPbI}_{3}$ is deposited on the ITO/glass compared to $\mathrm{MAPbI}_{3}$ on the bare glass (from which ITO coating was etched off before depositing the perovskite). Other evidences of intermixing of the perovskite components, e.g., iodine species, with the topmost ITO layers were also found. On the other hand, ITO prevents from the upward diffusion of admixtures, mainly alkali metals, from the bulk glass into the perovskite film.

ToF-SIMS confirms accumulation of $\mathrm{PbI}_{2}$ detected earlier by XRD and reveals intensive ablation of the organic component of perovskite from the film in the aged samples. The latter process is somewhat inhibited by ITO coating. On any substrate, early stages of degradation can explicitly be seen as blurring of the chemical interface between $\mathrm{MAPbI}_{3}$ and substrate. In case of the ITO/glass substrate, the indium species were detected throughout the degraded perovskite film, while the iodine species penetrate deeply into ITO. An interesting issue is the oxidation of perovskite layer [7], which was traced from the concentration of a large number of different oxygencontaining species. Except for the surface concentration jump, the oxidation front develops from the substrate side in all cases, so that non-oxidized intact perovskite, or its degraded residue after aging, remains close to the surface of the film, but not in the film bulk (cf. - [7]).

\section{Conclusion}

Efficiency of the archetypal cells with a hybrid $\mathrm{CuI} / \mathrm{C}_{60}$ heterojunction was improved by insertion of the hybrid perovskite $\mathrm{MAPbI}_{3}$. This is however not the case for the vacuum-deposited inorganic $\mathrm{CsPbI}_{3}$, which is not compatible with the adjacent components $\mathrm{CuI}$ and $\mathrm{C}_{60}$. In the inert atmosphere, the incident light in the range of $\sim 500 \mathrm{~nm}$ is responsible for the degradation of hybrid perovskite $\mathrm{MAPbI}_{3}$, removal of this factor extends the lifetime of this material. Degradation of $\mathrm{MAPbI}_{3}$ occurs not solely through segregation of $\mathrm{PbI}_{2}$ but also through transformation of $\mathrm{PbI}_{2}$ that has already existed in the intact perovskite structure. The latter transformation is probably incomplete and consists in the texturizing of $\mathrm{PbI} 2$ and in release of the metallic lead. Inorganic perovskite $\mathrm{CsPbI}_{3}$ is itself much more stable than its hybrid congener $\mathrm{MAPbI}_{3}$. However, its introduction into the multilayer cells with a given scheme/method is difficult, and the resulting devices show a very poor photovoltaic performance. Further optimization of the scheme and processing of the vacuum-only deposited photovoltaic cells incorporating inorganic perovskite is therefore a challenging issue.

\section{Acknowledgments}

This work was supported by RSF Grant \# 17-7910397.

\section{References}

[1] V.V. Travkin, P.A. Yunin, A.Y. Luk'yanov, P.A. Stuzhin, G.L. Pakhomov, Phys. Stat. Sol. A 214, 1700186 (2018).

[2] L.K. Ono, M.R. Leyden, S. Wang, Y. Qi, J. Mat. Chem. 4, 6655 (2016).

[3] M. Ahmad, G. Rehman, L. Ali, M. Shafiq, R. Iqbal, R. Ahmad, T. Khan, S. Jalali-Asadabadi, M. Maqbool, I. Ahmad, J. Alloys Comp. 705, 828 (2017).

[4] V.V. Travkin, A.Yu. Luk'yanov, M.N. Drozdov, E.A. Vopilkin, P.A. Yunin, G.L. Pakhomov, Appl. Surf. Sci. 390, 703 (2016).

[5] X. Tang, M. Brandl, B. May, I. Levchuk, Yi Hou, M. Richter, Hai-Wei Chen, Shi Chen, S. Kahmann, A. Osvet, F. Maier, H.-P. Steinrück, R. Hock, G.J. Matt, Ch.J. Brabec, J. Mater. Chem. A. 4, 15896 (2016).

[6] E. Lifshitz, M. Yassen, L. Bykov, R. Chaim, J. Phys. Chem. 99, 1245 (1995).

[7] N. Aristidou, C. Eames, I. Sanchez-Molina, XianGnan Bu, J. Kosco, M.S. Islam, S.A. Haque, Nature Comm. 8, 15218 (2017). 